\title{
Individual differences in sensory preferences for fat in model sweet dairy products
}

\author{
Adam Drewnowski * \\ Human Nutrition Program, School of Public Health M-5170, University of Michigan, Ann Arbor, \\ MI 48109-2029, USA
}

Sensory preferences for fat in foods are influenced both by subject characteristics and by the nature of the food stimulus. Hedonic responses to sugar/fat mixtures depend on strong interactions between perceptions of the two ingredients, including tactile as well as gustatory effects. Fat-containing liquid and solid foods, and dessert-type sugar/fat mixtures produce different hedonic response profiles. Body weight, weight history and the presence of eating disorders are associated with differences in hedonic responsiveness to fat among women. This inter-subject variability in hedonic response profiles mandates the use of novel techniques for data analysis. Conventional hedonic response curves mask between-subject differences. Individual patterns of hedonic responses to sugar/fat mixtures are better represented by frequency distributions of maximum hedonic responses or of individual optimal sugar-to-fat ratios.

The perception of fat in foods largely depends on texture felt in the mouth, and to a lesser degree on olfaction. For liquid dairy products, where fat is contained in emulsified globules, oral sensation of fat content is expressed in attributes such as stimulus thickness, smoothness, or creaminess (Cooper, 1987; Drewnowski, 1987a,b; Stanley and Taylor, this Issue). Fats also influence the mechanical and geometrical characteristics of solid foods (Brandt et al., 1963). Mechanical characteristics include hardness, adhesiveness and viscosity, while geometrical factors reflect the size, shape and orientation of food particles (e.g. coarse-grained or fibrous; cf. Tyle, this Issue). The so-called mouthfeel of solid foods generally involves the perception of moisture or fat in the oral cavity (e.g. wet, oily, greasy). Fats in solid foods facilitate mastication and swallowing (cf. Marshall, this Issue), and this oral processing also results in the sensory effects of fat changing with time.

* Tel: (313)-747-0208, Fax: (313)-764-5233.

0001-6918/93/\$06.00 (C) 1993 - Elsevier Science Publishers B.V. All rights reserved 
Clearly, the percepts that mediate oral assessment of fat content differ markedly between solid and liquid foods (Drewnowski at al., 1989).

Because fats are present in so many different food textures, no single attribute can fully describe the oral sensations of fat content or extract a common element in them. Instead, studies of the sensory effects of fat in foods have made use of a wide range of attribute terms to account for the diverse components of fat perception (Drewnowski, 1987a,b). According to Japanese studies (Yoshikawa et al., 1970), the key words describing the texture of foods were (in English translation): hard, soft, juicy, chewy, greasy, viscous, slippery, creamy, crisp, crunchy, and brittle. All these terms can be linked to the presence of fat.

Moreover, fat-like mouthfeel can be created using other substances, including modified starches and gums, microcrystalline cellulose and microparticulated protein. All of these mimic the tactile sensations from dairy fat, to some degree at least, and have found applications in the fat replacement products recently developed by the food industry (Drewnowski, 1992).

Thickeners and gels intensify the oral sensation of fatness (Drewnowski and Schwartz, 1990), while substances that reduce viscosity can result in judgments of lower fat content (Mela, 1988). Sugar, for example, may potentiate oral perception of fat in liquid dairy products: the addition of sugar to a low-fat dairy product such as skim milk raised stimulus viscosity and led to increased ratings of fatness and creaminess (Drewnowski and Greenwood, 1983).

Sugar can give solid food a denser and a more crystalline structure. Drewnowski and Schwartz (1990) showed that the addition of sugar to cake frostings effectively masked the oral perception of their fat content. In that study, 50 young women rated the sweetness and fat content of 15 model cake frostings, composed of sucrose (range 20-77\% wt/wt), polydextrose (a bland, partly metabolizable starch), unsalted butter (range $15-35 \% \mathrm{wt} / \mathrm{wt}$ ) and distilled water. As expected, sweetness intensity ratings varied only with sucrose levels. In contrast, fat contents ratings were a combined function of three ingredients: fat, polydextrose and water. Sugar masked the perception of fat content, most likely through altering texture: higher sugar contents led to reduced fatness and creaminess ratings at a constant level of fat.

This influence of sugar on the oral perception of fat may help explain why fat is often the invisible component of sweet, fat-rich desserts. Ice cream and chocolate are often thought of as sugary and therefore carbohydrate-rich foods. So-called 'carbohydrate cravings' (Paykel et al., 1973) appear to be largely preferences for chocolate, ice cream, pastries and other sweet confections and desserts that are rich in both sugar and fat.

That is to say, oral sensations generated by sugar and fat in familiar sugar /fat mixtures interact to determine the overall pleasantness of the food. This interactive nature of the sensory pleasure response has been observed 
using sweetened liquid dairy products containing different amounts of sugar and fat (Drewnowski and Greenwood, 1983; Drewnowski et al., 1985; 1987b) as well as solid sugar/fat mixtures (Drewnowski and Holden-Wiltse, 1992).

Drewnowski and Greenwood (1983), asked normal-weight adults to rate chilled mixtures of milk, cream and sugar for sweetness, fat content, and pleasantness. Respondents rated sweet stimuli at zero fat content (skim milk) and unsweetened dairy products relatively low in pleasantness. In contrast, hedonic ratings for sugar/fat mixtures resembling sweetened whipped cream were high. Similar results were subsequently obtained with other fat/sugar mixtures, such as sweetened cottage cheese and cream cheese, cake frostings and ice cream (Drewnowski et al., 1989; Drewnowski and Holden-Wiltse, 1992). For every stimulus, maximum average hedonic ratings did not depend on sugar only, but were a combined and synergistic function of both sugar and fat levels.

Published hedonic curves typically represent averaged group data. However, sensory preferences for sugar and fat in model dairy products show considerable individual variability. We investigated preferences for stimuli composed of sweetened creamy white cheese (fromage blanc) in a French group of bulimic women and in female controls (Drewnowski et al., 1987a). The fat content of the stimuli ranged from 0 to $7 \mathrm{~g}$ per $100 \mathrm{~g}$ of product, while sugar content ranged from 1 to $40 \%$ weight/weight. Bulimic women as a group showed higher hedonic optima for sugar than did control women, and a sharper decline in average preference with increasing fat content (Fig. 1.). However, these averaged pleasantness ratings provide no indication of inter-subject variability. Consequently, further analyses addressed the hedonic response profiles of individual women. Frequency distributions of maximum hedonic scores as a function of stimulus sugar and fat content are shown in Fig. 2, separately for bulimic women and normal controls. More bulimic patients preferred intensely sweet stimuli ( $20 \%$ sucrose) than did controls. In contrast, control subjects overwhelmingly preferred stimuli with a higher fat content: their modal maximum response was for stimuli containing $7 \mathrm{~g}$ fat per $100 \mathrm{~g}$ of product. It should be noted, however, that there was considerable overlap between the two groups of women. It was impossible to assign a woman to a diagnostic category solely on the basis of her sensory preferences for sugar or fat (Drewnowski et al., 1987a).

The individual differences in sensory preference may be related also to body weight status to some extent. Drewnowski et al. (1985) found that massively obese women gave highest pleasantness ratings to stimuli containing $34 \%$ fat but only $4 \%$ sugar, while normal-weight women preferred stimuli containing approximately $20 \%$ fat and $9 \%$ sugar. The effect of sugar on preference was strongly modulated by fat content of the material. Obese women disliked $10 \%$ sucrose in skim milk, but liked the same concentration of sucrose when presented in heavy cream. 


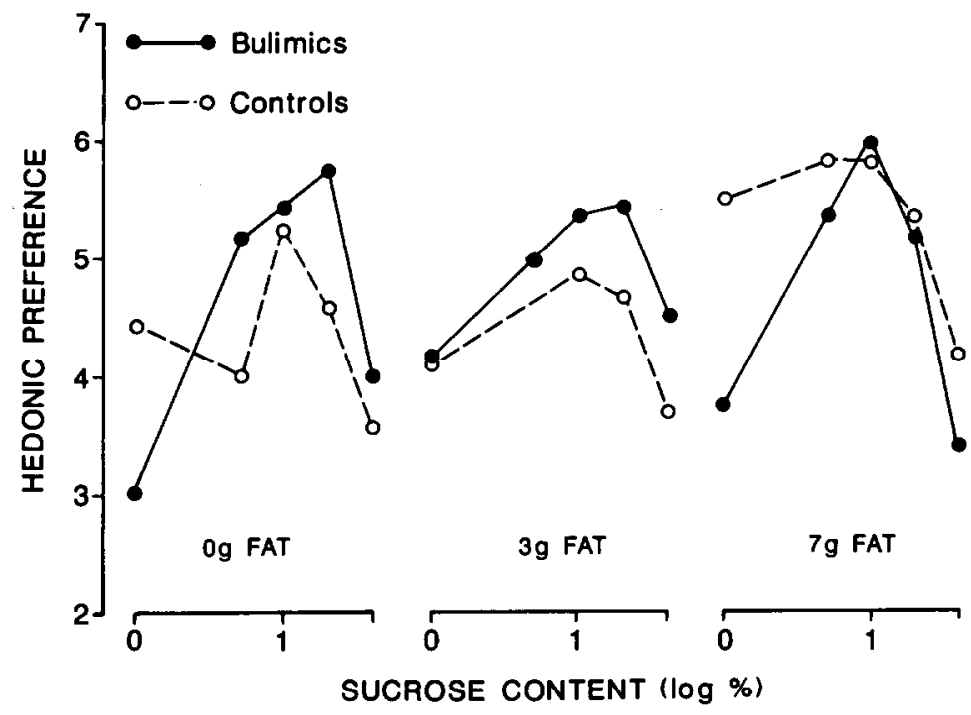

Fig. 1. Mean hedonic ratings by bulimic and control women, as a function of sucrose content for each type of dairy product.

In another study (Drewnowski et al., 1987b), young women hospitalized for anorexia or bulimia nervosa selected sweetened dairy products that were relatively high in sugar $(13 \%)$ but were extremely low in fat. Their sensory functioning, as assessed by the ratings of sweetness and fat content, was not altered. A strong dislike for fat in foods seems to be a characteristic symptom of anorexia nervosa. While past studies on anorectic women have invoked the concept of 'carbohydrate phobia', fat avoidance seems to be closer to the mark.
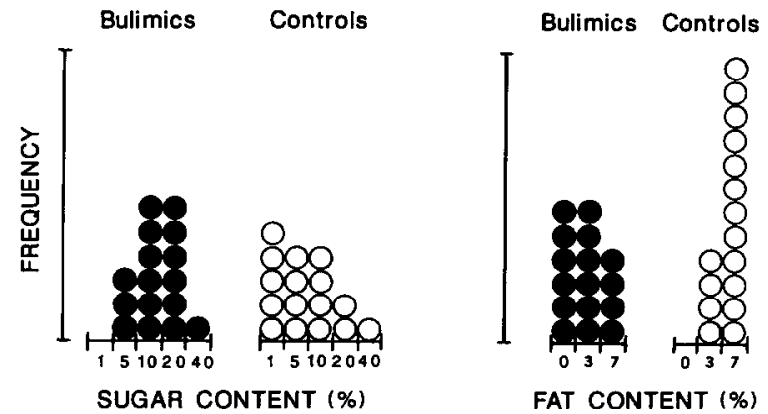

Fig. 2. Frequency distribution of maximum hedonic scores from women with and without bulimia, as a function of stimulus sugar and fat content (\% wt $/ \mathrm{wt})$. 


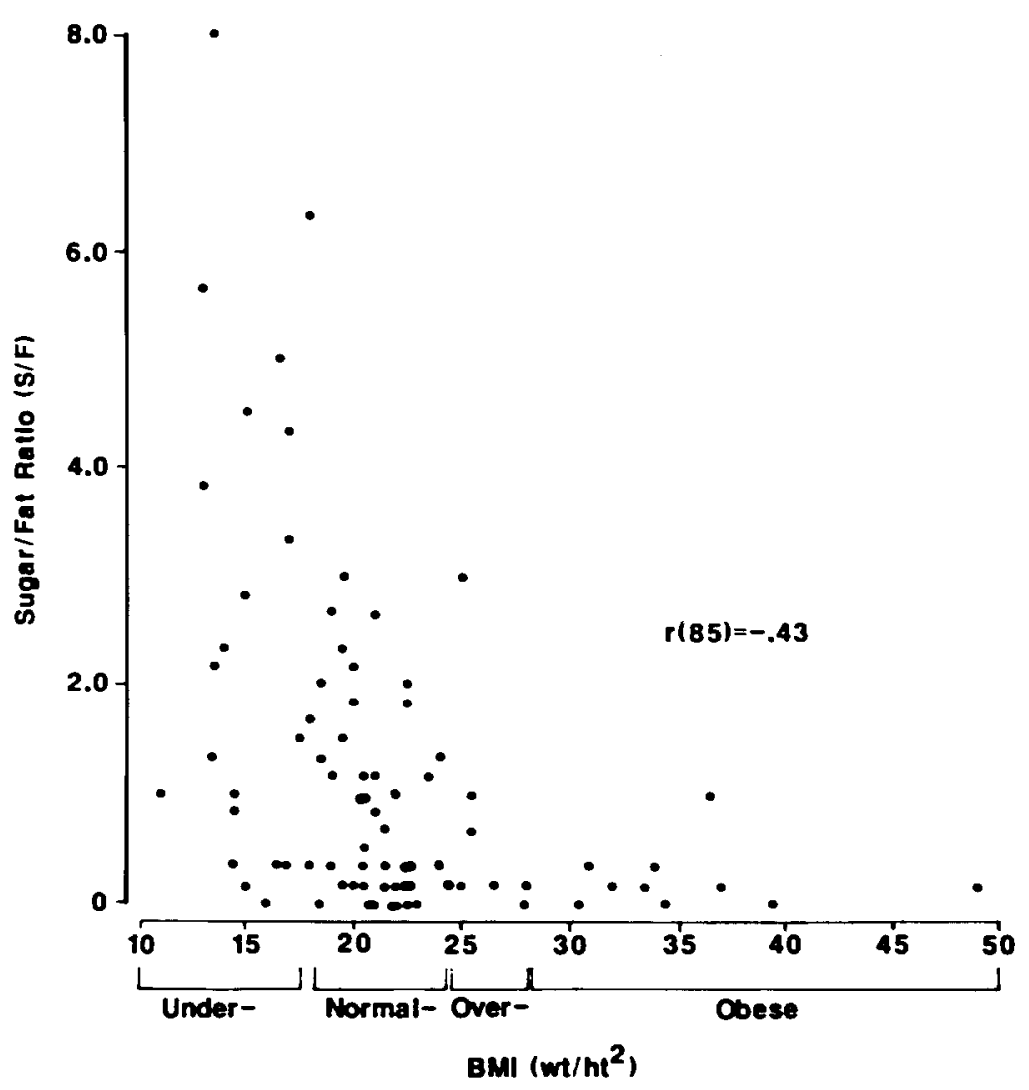

Fig. 3. Relationship between the optimally preferred sugar/fat ratios by weight and body mass indiccs $\left(\mathrm{kg} / \mathrm{m}^{2}\right)$ of underweight, normal-weight, and obese women.

Sensory preferences for sugar relative to fat in sweet dairy products appear to vary inversely with body fatness. In studies of women at the extremes of body weight (Drewnowski et al., 1985; 1987b), the most preferred sugar /fat ratio was inversely related to the body mass index (BMI: $\mathrm{kg}$ body weight $/ \mathrm{m}^{2}$ body mass). While emaciated anorectic women selected the sweetest taste stimuli, obese women (BMI $>40)$ tended to select those stimuli that were rich in fat. This finding was recently replicated in a study showing that sensory preferences for fat in foods were positively correlated with the subjects' percent body fat (Mela and Sacchetti, 1991).

However, body weight is only one of many factors that may relate to individual preferences for fat in foods. The relationship between BMI and individuals' sensory preferences for sugar relative to fat, shown in Fig. 3, while statistically significant, accounted for only $18 \%$ of the variance. 
One other factor affecting sensory preferences for food materials may be recent weight history and especially the presence of weight cycling or 'yo-yo' dieting. Drewnowski and Holden-Wiltse (1992) categorized obese women as weight cyclers or non-cyclers on the basis of their past fluctuations in body weight. In that study, 37 obese women rated the pleasantnesses of a range of sucrose solutions as well as of nine specially prepared ice creams of varying sugar and fat content. The responses to sweet solutions were the same for the two groups but weight cyclers showed significantly greater preferences for the ice creams than did non-cyclers. The weight cyclers also rated sweet desserts higher on a food preference questionnaire than did non-cyclers.

Other factors affecting preferences for sugar/fat mixtures may include concerns, especially among dieters, about the consumption of sugar and fat. Such concerns are less likely to affect the ratings of stimuli that are clearly different from real foods. Sucrose solutions in distilled water have been regarded as giving relatively bias-free responses but their usefulness is limited because the responses to such stimuli do not necessarily predict food-related behaviors or the consumption of real foods. Model systems resembling real foods, for example sweetened dairy products, are more likely to be affected by health-related biases. The dislike for sugar/fat mixtures observed among patients suffering from anorexia nervosa is sometimes associated with extremely high ratings of sweetness or fat content, not attributable to increased gustatory or tactile sensitivity. Other examples of health bias are seen among normal-weight young women dieters, who routinely profess a dislike of sweet solutions.

The pleasure response to solid sugar/fat mixtures does not depend on an accurate sensory assessment of fat content. In one study (Drewnowski et al., 1989), 25 subjects rated the sweetness, creaminess and fat content as well as the acceptability of a range of liquid and solid dairy products containing both sugar and fat. Liquid stimuli included sweetened skim milk, whole milk, half and half, and heavy cream, while the solids were sweetened blends of cottage cheese and cream cheese, spread on slices of white bread. Ratings of sweetness and fat content were substantially lower for the solids than they were for liquid foods. Despite instructions, some assessors were unable to track the increasing fat content of the solid foods. In contrast, pleasantness ratings for the two sets of stimuli were not appreciably different. While the subjccts optimally preferred similar levels of sugar in both liquid and solid foods, they selected higher levels of fat in solids than in liquids (Drewnowski et al., 1989).

Elevated preferences for fat-rich solid foods in the absence of accurate assessment of fat content suggest that food acceptability ratings need not be analytical in nature, and do not depend on consciousness of the amount of fat in the food samples. Furthermore, the most preferred percentage of fat is likely to vary from one food system to another. 
In addition, individual differences in patterns of sensory preference are a function of a person's body weight, percent body fat and weight history, and can be expected to relate to attitudes and beliefs regarding health and dieting, and past history of exposure to foods (Tuorila and Pangborn, 1988). The response profiles of individual overweight subjects to sugar/fat mixtures predict their preferences for some sweet high-fat foods. Conversely, low preferences for dairy products in anorexia nervosa were consistent with the avoidance of most high-fat foods, notably milk and meat, reported by such patients. Thus, preference profiles for model sweet dairy products may provide a useful experimental marker of individual responsiveness to a high-fat diet.

\section{References}

Brandt, M.A., E.Z. Skinner and J.A. Coleman, 1963. Texture profile method. Journal of Food Science 28, 404-409.

Cooper, H.R., 1987. 'Texture in dairy products and its sensory evaluation'. In: H.R. Moskowitz (Ed.), Food texture (pp. 217-250). New York: Marcel Dekker.

Drewnowski, A., 1987a. 'Fats and food texture: Sensory and hedonic evaluations'. In: H.R. Moskowitz (Ed.), Food texture. New York: Marcel Dekker.

Drewnowski, A., 1987b. 'Fats and food acceptance: Sensory, hedonic and attitudinal aspects'. In: J. Solms, D.A. Booth, R.M. Pangborn and O. Raunhardt (Eds.), Food acceptance and nutrition. New York Academic Press.

Drewnowski, A., 1987c. 'Sweetness and obesity'. In: J. Dobbing (Ed.), Sweetness. New York: Springer-Verlag.

Drewnowski, A., 1992. Sensory properties of fats and fat replacements. Nutrition Reviews 50 , $17-20$.

Drewnowski, A., F. Bellisle, P. Aimez and B. Remy, 1987a. Taste and bulimia. Physiology \& Behavior 41, 621-626.

Drewnowski, A., J.D. Brunzell, K. Sande, P.H. Iverius and M.R.C. Greenwood, 1985. Sweet tooth reconsidered: Taste preferences in human obesity. Physiology \& Behavior 35, 617-622.

Drewnowski, A. and M.R.C. Greenwood, 1983. Cream and sugar: Human preferences for high-fat foods. Physiology \& Behavior 30, 629-633.

Drewnowski, A., K.A. Halmi, B. Pierce, J. Gibbs and G.P. Smith, 1987b. Taste and eating disorders. American Journal of Clinical Nutrition 46, 442-450.

Drewnowski, A. and J. Holden-Wiltse, 1992. Taste responses and food preferences in obese women: Effects of weight cycling. International Journal of Obesity 16, 639-648.

Drewnowski, A. and M. Schwartz, 1990. Invisible fats: Sensory assessment of sugar/fat mixtures. Appetite 14, 203-217.

Drewnowski, A., E.E. Shrager, C. Lipsky, E. Stellar and M.R.C. Greenwood, 1989. Sugar and fat: Sensory and hedonic evaluations of liquid and solid foods. Physiology \& Behavior 45, $177-183$.

Marshall, R.J., 1993. Physicochemical properties accounting for cheese texture. Acta Psychologica 84, 69-77 (this Issue).

Mela, D.J., 1993. Sensory assessment of fat content in fluid dairy products. Appetite 10, 37-44.

Mela, D.J. and D.A. Sacchetti, 1991. Sensory preferences for fats: Relationships with diet and body composition. American Journal of Clinical Nutrition 53, 908-915. 
Paykel, E.S., P.S. Mueller and P.M. de la Vergne, 1973. Amitryntiline, weight gain and carbohydrate craving: A side effect. British Journal of Psychiatry 125, 501-507.

Stanley, N.L. and L.J. Taylor, 1993. Rheological basis of oral characteristics of fluid and semi-solid foods: A review. Acta Psychologica 84, 79-92 (this Issue).

Tuorila, H. and R.M. Pangborn, 1988. Prediction of reported consumption of selected fat-containing foods. Appetite 11, 81-95.

Tyle, P., 1993. Effect of size, shape and hardness of particles in suspension on oral texture and palatability. Acta Psychologica 84, 111-118 (this Issue).

Yoshikawa, S., S. Nishumaru and M. Yoshida, 1970. Collection and classification of words for description in food texture, III: Classification by multivariate analysis. Journal of Texture Studies 1, 452-463. 